\title{
A Simple Proof of Fast Polarization
}

\author{
Ido Tal \\ Department of Electrical Engineering \\ Technion - Haifa 32000, Israel \\ E-mail: idotal@ee.technion.ac.il
}

\begin{abstract}
Fast polarization is an important and useful property of polar codes. It was proved for the binary polarizing $2 \times 2$ kernel by Arıkan and Telatar. The proof was later generalized by Şaşoğlu. We give a simplified proof.
\end{abstract}

Index Terms

polar codes, fast polarization

\section{INTRODUCTION}

Polar codes are a novel family of error correcting codes, invented by Arıkan [1]. The seminal definitions and assumptions in [1] were soon expanded and generalized. Key to almost all the results involving polar codes is the concept of fast polarization. The essence of fast polarization is the phenomenon stated in the following lemma. The lemma was used implicitly by Korada, Şaşoğlu, and Urbanke [2] proof of Theorem 11], and is a generalization of a result by Arıkan and Telatar [3, Theorem 3]. Its explicit formulation and full proof are due to Şaşoğlu [4, Lemma 5.9].

Lemma 1: Let $B_{0}, B_{1}, \ldots$ be an i.i.d. process where $B_{0}$ is uniformly distributed over $\{1,2, \ldots, \ell\}$. Let $Z_{0}, Z_{1}, \ldots$ be a $[0,1]$-valued random process where

$$
Z_{m+1} \leq K \cdot Z_{m}^{D_{t}}, \quad \text { whenever } B_{m}=t
$$

We assume $K \geq 1$ and $D_{1}, D_{2}, \ldots, D_{\ell}>0$. Suppose also that $Z_{m}$ converges almost surely to a $\{0,1\}$-valued random variable $Z_{\infty}$. Then, for any

$$
0<\beta<E \triangleq \frac{1}{\ell} \sum_{t=1}^{\ell} \log _{\ell} D_{t}
$$

we have

$$
\lim _{m \rightarrow \infty} \operatorname{Pr}\left[Z_{m} \leq 2^{-\ell^{\beta \cdot m}}\right]=\operatorname{Pr}\left[Z_{\infty}=0\right] .
$$

The lemma is used to prove that the Bhattacharyya parameter associated with a random variable that underwent polarization (for example, a synthesized channel) polarizes to 0 at a rate faster than polynomial [4, Theorem 5.4]. A similar claim holds in the case of polarization of the Bhattacharyya parameter to 1 [5, Theorem 16].

The original proof [4, Lemma 5.9] of Lemma 1 is somewhat involved. To summarize, if $K$ were equal to 1, the proof would follow almost directly from the strong law of large numbers. However, for $K>1$, a sequence 
of bootstrapping arguments is applied. That is, a current bound on the rate of convergence of $Z_{m}$ to 0 is used to derive a stronger bound, and the process is repeated.

The main aim of this paper is to give a simpler proof of Lemma 1. Thus, we hopefully give insight into the simple mechanics that are at play. Our simpler proof also leads to a stronger result. That is, we will prove the following, which implies Lemma 1.

Lemma 2: Let $\left\{B_{m}\right\}_{m=0}^{\infty},\left\{Z_{m}\right\}_{m=0}^{\infty}, K$, and $E$ be as in Lemma 1 Then, for $0<\beta<E$,

$$
\lim _{m_{0} \rightarrow \infty} \operatorname{Pr}\left[Z_{m} \leq 2^{-\ell^{\beta \cdot m}} \text { for all } m \geq m_{0}\right]=\operatorname{Pr}\left[Z_{\infty}=0\right]
$$

Note that Lemma 2] has an "almost sure flavor" [6, page 69, Equation (2)], while Lemma 1 has an "in probability flavor" [6, page 70, Equation (5)]. We prove Lemma 2 in Section $\coprod$ and show that it implies Lemma 1 in Section III

\section{Proof of Lemma 2}

Let $\epsilon_{a}, \epsilon_{b}>0$ and $m_{a}<m_{b}$ be given constants, specified towards the end. We now define three events, denoted $A, B$, and $C$.

$$
\begin{array}{ll}
A: & \left|Z_{m}-Z_{\infty}\right| \leq \epsilon_{a}, \quad \text { for all } m \geq m_{a} . \\
B: & \left|\frac{\left|\left\{m_{a} \leq i<m: B_{i}=t\right\}\right|}{m-m_{a}}-\frac{1}{\ell}\right| \leq \epsilon_{b}, \quad \text { for all } m \geq m_{b} \text { and } 1 \leq t \leq \ell . \\
C: & Z_{\infty}=0 .
\end{array}
$$

Recall that the $Z_{m}$ converge almost surely to $Z_{\infty}$. Thus, essentially by definition (see [6, Theorem 4.1.1.]), we have for any fixed $\epsilon_{a}>0$ that

$$
\lim _{m_{a} \rightarrow \infty} \operatorname{Pr}[A]=1
$$

Note that event $B$ is concerned with the frequency of $t$ in the subsequence of i.i.d. random variables $B_{m_{a}}, B_{m_{a}+1}, \ldots, B_{m-1}$, which are uniform over $\{1,2, \ldots, \ell\}$. Thus, by the strong law of large numbers 16 , Theorem 5.4.2.], we have for any fixed $\epsilon_{b}$ and $m_{a}$ that

$$
\lim _{m_{b} \rightarrow \infty} \operatorname{Pr}[B]=1
$$

We deduce that for any $\delta_{a}, \delta_{b}>0$ there exist $m_{a}<m_{b}$ such that

$$
\operatorname{Pr}[A] \geq 1-\delta_{a}
$$

and

$$
\operatorname{Pr}[B] \geq 1-\delta_{b}
$$

Hence,

$$
\operatorname{Pr}[A \cap B \cap C] \geq \operatorname{Pr}\left[Z_{\infty}=0\right]-\delta_{a}-\delta_{b}
$$

${ }^{1}$ The strong law of large numbers is applied $\ell$ times. Each application is with respect to the indicators $B_{i}=t$, where $1 \leq t \leq \ell$. As before, we use [6 Theorem 4.1.1.]. 
Let us see what the event $A \cap B \cap C$ implies. For fixed $0<\epsilon_{a}, \epsilon_{b}, \delta_{a}, \delta_{b}<1$, let $m_{a}$ and $m_{b}$ be as above. Define the shorthand

$$
\theta \triangleq-\log _{\epsilon_{a}} K
$$

Note that $\theta$ is non-negative, and approaches 0 as $\epsilon_{a}$ approaches 0 . By the definition of the events $A$ and $C$, we have that $Z_{m} \leq \epsilon_{a}$ when $m \geq m_{a}$. Thus, $K \leq Z_{m}^{-\theta}$ when $m \geq m_{a}$. Hence, we simplify (1) to

$$
Z_{m+1} \leq Z_{m}^{D_{t}-\theta}, \quad \text { whenever } m \geq m_{a} \text { and } B_{m}=t .
$$

The above equation is the heart of the proof: we have effectively managed to "make $K$ equal 1" - the simple case discussed earlier. We have "paid" for this simplification by having the exponents be $D_{t}-\theta$ instead of the original $D_{t}$. However, since $\theta$ can be made arbitrarily close to 0 , this will not be a problem. Essentially, all that remains is some simple algebra, followed by taking the relevant parameters small/large enough. We do this now.

Let us assume $\epsilon_{a}$ is small enough such that $D_{t}-\theta>0$ for all $1 \leq t \leq \ell$ and that $\epsilon_{b}<1 / \ell$. Recall also that $Z_{m_{a}} \in[0,1]$. Combining (10) with event $B$, we deduce that for all $m \geq m_{b}$,

$$
Z_{m} \leq Z_{m_{a}}^{\prod_{t=1}^{\ell}\left(D_{t}-\theta\right)^{\left(m-m_{a}\right) \cdot\left(1 / \ell \pm \epsilon_{b}\right)}}
$$

where the above " \pm " notation is in fact a function of $t$, defined as

$$
\pm \triangleq \begin{cases}+ & \text { if } D_{t}-\theta \leq 1 \\ - & \text { otherwise. }\end{cases}
$$

By the definition of event $A$, we have that $Z_{m_{a}} \leq \epsilon_{a}$. We will further assume that $\epsilon_{a} \leq 1 / 2$. Hence, (11) simplifies to the claim that for all $m \geq m_{b}$,

$$
Z_{m} \leq 2^{-\prod_{t=1}^{\ell}\left(D_{t}-\theta\right)^{\left(m-m_{a}\right) \cdot\left(1 / \ell \pm \epsilon_{b}\right)}}=2^{-\ell^{(E-\Delta) m}}
$$

where

$$
\Delta=\sum_{t=1}^{\ell} \frac{1}{\ell} \log _{\ell}\left(\frac{D_{t}}{D_{t}-\theta}\right)-\sum_{t=1}^{\ell} \pm \epsilon_{b} \log _{\ell}\left(D_{t}-\theta\right)+\sum_{t=1}^{\ell} \frac{m_{a}}{m}\left(\frac{1}{\ell} \pm \epsilon_{b}\right) \log _{\ell}\left(D_{t}-\theta\right) .
$$

In light of (3), our goal is to show that for a given $\beta<E$ and $\delta_{a}, \delta_{b}>0$, we can choose $m_{a}<m_{b}$, and $\epsilon_{a}, \epsilon_{b}>0$ as above such that $\Delta<E-\beta$. We do this by showing that each of the three sums in (13) can be made smaller than $(E-\beta) / 3$. Recalling that $\theta$ goes to 0 as $\epsilon_{a}$ tends to 0 , we deduce that the first sum can be made smaller than $(E-\beta) / 3$ by taking $\epsilon_{a}$ small enough. Similarly, we can make the second sum smaller than $(E-\beta) / 3$ by taking $\epsilon_{b}$ small enough. For the third sum, we first fix $m_{a}$ large enough such that (7) holds (note that event $A$ is a function of $\epsilon_{a}$, which is by now fixed). Lastly, we take $m_{b}$ large enough such that the third sum is smaller than $(E-\beta) / 3$ for all $m \geq m_{b}$, and $(8)$ holds (again, note that event $B$ is a function of $m_{a}$ and $\epsilon_{b}$, which have been fixed).

Recall that our aim is to prove (3). We deduce from (9), (12), and the above paragraph that for all $\delta_{a}, \delta_{b}>0$ and $0<\beta<E$,

$$
\lim _{m_{0} \rightarrow \infty} \operatorname{Pr} \overbrace{\left.Z_{m} \leq 2^{-\ell^{\beta \cdot m}} \text { for all } m \geq m_{0}\right]}^{D} \geq \operatorname{Pr}\left[Z_{\infty}=0\right]-\delta_{a}-\delta_{b} .
$$


Indeed, we have just proved that for the parameters fixed as above, $A \cap B \cap C$ implies $D$, for $m_{0}=m_{b}$. Since we are taking the limit of a strictly increasing sequence, the assertion follows (and the limit exists, since the sequence is bounded).

Since the above inequality holds for all $\delta_{a}, \delta_{b}>0$, it must also hold for $\delta_{a}=\delta_{b}=0$. Thus, all that remains is to prove that

$$
\lim _{m_{0} \rightarrow \infty} \operatorname{Pr}\left[Z_{m} \leq 2^{-\ell^{\beta \cdot m}} \text { for all } m \geq m_{0}\right] \leq \operatorname{Pr}\left[Z_{\infty}=0\right]
$$

Assume to the contrary that there exists $0<\beta<E$ and $m_{0}$ such that

$$
\operatorname{Pr}\left[Z_{m} \leq 2^{-\ell^{\beta \cdot m}} \text { for all } m \geq m_{0}\right]>\operatorname{Pr}\left[Z_{\infty}=0\right]
$$

Clearly, this implies that

$$
\operatorname{Pr}\left[\lim _{m \rightarrow \infty} Z_{m}=0\right]>\operatorname{Pr}\left[Z_{\infty}=0\right]
$$

Hence,

$$
\operatorname{Pr}\left[\lim _{m \rightarrow \infty} Z_{m}=Z_{\infty}\right]<1
$$

contradicting that fact that the sequence $Z_{m}$ converges almost surely to $Z_{\infty}$.

\section{PROOF OF LEMMa 1}

We now explain why Lemma 2 implies Lemma 1, That is, why (3) implies (2). Clearly, (3) implies

$$
\liminf _{m \rightarrow \infty} \operatorname{Pr}\left[Z_{m} \leq 2^{-\ell^{\beta \cdot m}}\right] \geq \operatorname{Pr}\left[Z_{\infty}=0\right]
$$

Thus, the claim will follow if we prove that

$$
\limsup _{m \rightarrow \infty} \operatorname{Pr}\left[Z_{m} \leq 2^{-\ell^{\beta \cdot m}}\right] \leq \operatorname{Pr}\left[Z_{\infty}=0\right] .
$$

Assume to the contrary that there exists $0<\beta<E$ such that

$$
\limsup _{m \rightarrow \infty} \operatorname{Pr}\left[Z_{m} \leq 2^{-\ell^{\beta \cdot m}}\right]>\operatorname{Pr}\left[Z_{\infty}=0\right]
$$

The above implies that the $Z_{m}$ cannot converge in probability to $Z_{\infty}$ [6, page 70, Equation (5)]. This contradicts the fact that almost sure convergence implies convergence in probability [6, Theorem 4.2].

\section{REFERENCES}

[1] E. Arıkan, "Channel polarization: A method for constructing capacity-achieving codes for symmetric binary-input memoryless channels," IEEE Trans. Inform. Theory, vol. 55, no. 7, pp. 3051-3073, July 2009.

[2] S. B. Korada, E. Şaşoğlu, and R. Urbanke, "Polar codes: Characterization of exponent, bounds, and constructions," IEEE Trans. Inform. Theory, vol. 56, no. 12, pp. 6253-6264, December 2010.

[3] E. Arıkan and E. Telatar, "On the rate of channel polarization," in Proc. IEEE Int'l Symp. Inform. Theory (ISIT'2009), Seoul, South Korea, 2009, pp. 1493-1495.

[4] E. Şaşoğlu, "Polarization and polar codes," in Found. and Trends in Commun. and Inform. Theory, vol. 8, no. 4, 2012 , pp. $259-381$.

[5] S. B. Korada and R. Urbanke, "Polar codes are optimal for lossy source coding," IEEE Trans. Inform. Theory, vol. 56, no. 4, pp. 1751-1768, April 2010.

[6] K. L. Chung, A Course in Probability Theory, 3rd ed. San Diego: Academic Press, 2001. 